de la turbine, ưn joint étanche. Dans l'espëce, ce joint est constittué par deux bagưes en graphite, ne nécessitant pas de graissage, et frottant contre l'arbre. Pour assurer, en outre, une étancliéité plus parfaite, et éviter teute rentrée d'air au condenseur, on envole un jet de vapeur dans cette sorte de presscétoupe. Un déflecteur et une cuvetie avec xuyau de vidange empêchent que la faible quantité de cette vapeur qui se condense ne glisse le long de l'arbre et, par suite, ne vienne se mélanger à l'huile de graissage du palier de guidage supérieur.

Les pressions adoptées pour le graissage à l'huile sonf les mểmes que celles adoptées pour le graissage à l'eau, et les précautions à prendre sont également les mêmes.

D"une manière générale, on installe toujours deux pompes suffisantes pour assurer chacune le graissiage du rrombre total d'unités constiturant l'usine, l'une de ces pompes devant servit de secour's en cas davarie à celle' en service. Le tuyau collecteur de refoulement de ces pompes est souvent double, et, dans ce cas, la tuyauterie est prévue de manière que chaque pompe puisse débiter dans: l'un ou l'autre collecteur, ceux-ci étant également reliés l'un à l'autre au pirot de chaque turbrne. Enfin, un accumulateur dean ou d'huile so is pression est également préru, branché sur le collecteur de refoulement des pompes de graissage, pour assurer le service en cas d'accident, pendant le temps nécessaire à la mise en fonctionnement de la pompe de secours.

Pour parer au cas où une pompe de graissage viendrait à. s'arrêter, par exemple par suite de la rupture d'un fusible du moteur de commande, les accumulateurs sont généralement munis d.un avertisseur sonore prévenant le personnel du moment où ils commencent à fonctionner.

Les turbines à axe vertical ayant été l'objet de crítiques ayant trait au pivot, M. Postel-Vinay tient à sígniter que, mểme au $c$. is où l: graissage viendrait à manquer complètement, il ne parait pas devoir en résulter d'avarie grave. Le fait a été expérimenté à titre dessai en Amárique, sur une turbine de 5000 kilowatts, tournant à 500 tours, et dont le poids des masses en mouvement était d'environ 35 tonnes Alors que cette machine était en pleine vitesse, on a arrèté l'arrivée d'huile au pirot et coupé la vapeur; après stoppage de la turbine (qui s'est produit en quelques minutes, a lieu de.3o minutes, temps normal) la crapaudine a été dénrontée et les grains ont été verifis. Ils ne portaient que quelques traces de grippement insignifiantes, et ont été remontés tels quels sarns aucune rectification.

M. Postel-Vinay a eu l'occasion, ir y a un an, de vérifier lui-même ce fait, lors de la misé en route d'une unité de 1500 H.P. Par deux fors, accidentellement, le graissage a manqué au pivot, ators que la turbine tournait à $\mathrm{r} 500$ tours. On a immédiatem ent coupé la varpeur, l'arrêt s'est également produit en quelques minutes et les deux fois, après véritication des faces portantes; les grans ont été remis en place sans avoir subi aucune réparation. Or, depuis: um an, certe turbine assure un service public régulier sans qu'll y ait ea aucun inconvénient du fait du pivot.

M. Postel-Vinay ajoute que, dans le cas du graissage par huile, c'est la même huile qui ressert presque indéfiniment et qu'il suffit de compinser les scules partes qui peuvent se produire par fuites dans les tuyauteries. Cela suftit à prouver qu'il n'y a pas usure des surfaces en contact.

Ces quelques considérations tendent à établir que le pivor en question n'est pas un organe aussi délicat: qu'on pourrair lé croire.

\section{INVENTIONS NOUVELLES}

Procédé pour faire varier la vitesse des moteurs asynchromes. - Brevet. no 361 . I 20. Société Thomson-Houston, le 27 décembre. 1905 .

On sait que les moteurs asynchromes ordintaires, ne peưvent avoir une vitesse réglable avec bon rendement à toutes viteses.

Ee procédé qứ consiste à intercaler des résistances. diars' les cireuits du rotor procure bren une bitisse de vitesse, nuais au détrintent d'une consom.nation d'énergíe supplémentaire proportionnelle à cette baisse de ritesse.

Un procédé qui permet d'obtenfr certaines variations de vitesse consiste dans l"emploi des moteurs à nombre de pốles variable dont il existe un certain nombre de realisations ; rous citerons entre autres le brevet français no 353.99 I du 5 mai 1905 . On obtient' ainsi un certain nombre de vitesses de régime avec bon rendement.

Un autre procédé bien comnu corrsiste à munir le moteur d'un collecteur Gramme, nais le moteur devient beaucoup plus cotiteax, et présente de plus un certaín nombre d ínconvénients:

Ih arrive quelquefớis, et c'est ce que vise spécialement cette invention; que Pon a besoin dé régler la vitesse seulement dans dés linnites assez peu étendues, par exemple de l'ardre de 20 poưr roo. Ceci-se prés nte, entre autrés, lorsque l'on vét actionner des ventilatenrs ou de's pompes, et que l'on désire en fứre varier' le débit ou la pressiont tout en gardant un bont rendement.
Une première réalisation de ce problëm 2 est visée dans le breve francais no $358.098 \mathrm{da} 29$ septembre 1905 , où "ơn ferme les circuilts du rotur sur un transformateur à champ tournant avez collecteur.

On connaît en outre le système dés moteurs en cascade qui permet d'obtenir avec ieux moteurs deux vitesses avec bon rendement. Ce système a été appliqué aussi avec les deux moteurs à nombre de pôles variable; et alors on peut obtenir beaucoup d: vitesses aveo bon rendement.

Ces systèmes ont différents inconvénients : les uns exigent l'emploi d'un collecteur Gramme; les autres, à nombre de pôles variable, s@nt assez compliqués comme réalisation lorsque l'on a plus de deux ou trois vitesses, et que de plus les moteurs dolvent êtr une ligne à haute tension.

La présente invention a pour but de réaliser l'ajustement de la vitesse dans de certaines limites tout en éliminant les inconvénients dont il est parlé ci-dessus. Elle consiste en un moteur principal, de construction tout à fait normale, que l'on peut faire facilement à haute tension. Ce moteur à nombre de pôtes constant tournerait à vitesse de régime constante s'it était seule. Mais son rotor, au lieu d'ètre mis en court-circuit directement, peut êtré fermé sur le stator d'un moteur auxiliaire ă nombre de pốles variable. Le stator de ce moteur est, comme le votor du moteur principal, à basse terision. Son bobinage avec nombre de pốles variable est donc très tacile. Qưant au: rotor de ce moteur auxiliaire', il peure être bobiné atrec bagues, ou en cage d'écureulil. Le démarrage pourra se faire en intercalant des résistances progressivement supprimées entre le rotor du moteur principal et le rotor du moteur auxiliaire. Les deux moteurs ont leurs arbres liés mécaniquement, soit directement, soit par engrenages ou tout autre procédé connu. Quant à la purssance du moreur auxiliaire, elle sera taible à côté de celle du moteur principal, cár Ia puissance qu'il aura a fournir sera seulement cellè qu'on auraít éu à depenser dans des rhéostats si l'on avait fait la variation de vitesse par ce procédé. Ce moteur auxiliaire joue donc le rôle de récupérateur.

Par exemple, supposons ur ventilateur absorbant 200 chevaux à 200 tours par minute, vitesse qui doit ètre abaissée à I 65 tours par minute avec 4 vitesses de régime sans résistances, et qu'on dispose de courant triphasé à la fréquence de 50 par seconde. On fera un moteur principal à ?o pôles; on aura ainsí avec le rotor du moteur principal en court-circuit une vitesse de 200 tours par minute.

Le moteur à nombre de pôles variable sera bobiné pour 2,4 ou 6 pôles par le changement- de positron d'tru commutateur; le bobinage et la disposition générale pourraient être selon le brevet français no 353.99 d du 6 mai 1005

Les deux arbres sernnt liés soltidirement pour tourner à même vitesse. Si le rotor du moteur principal est fermé sur le stator du moteur auxiliaire avec la combina son à $z$ pôles, on obtiendra la vitesse de 187 tours par minute. Si le rotor du mateur pr ncipal est fermé sur le stator du moteur auxiliaire avec la combinaison à 4 póles, on obtiendra la vitesse de 177 tours par minute. Si le rotor du moteur principal est fermé sur le stator du moteur auxiliaire avec la combinaison ä 6 pôles, on obtiendra 866 tours par minute.

La puissance du moteur auxiliaire sera proportion melle à la baisse de vitesse obtenue, soit approximativement dans les trois cas, 6,5; 11,5 et 17 pur roo déla puissance du moteur prineipal. Il est à remarquer que, dars la plupart des cas, la puissance nécessaire baisiera avec la vitesse, et que si l'on a besoin de 200 chevaux à 200 tours, il suffira, par exemple, de 180,160 et r 40 chevaux aux trois autres vitesses; de sorte que le moteur auxiliaire doit faire environ 12 , I 8 et 24 chevaux, c'est-à-dire moins de un huitième de la puissance normale da moteur principal.

RÉsumé.- La présente invention a pour but de téaliser des vitesses variables dans une certaine limite en se servant de courants alternatifs, simple ou polyphasé, par la combinaison: 10 D'un moteur asynchrone normal de construction tout $\grave{a}$ fait ordinaire; d'un moteur asynchrone auxiliaire à nombre de pôles variable, bobiné selon les procédés connus, et jouant le rôle de récupérateur. Le stator du moteur auxiliaire est fermé sur le rotor du moteur principal.

\section{INFORMATIONSS DIVERSES}

\section{Statistique des stations centrales suisses}

L'Association suisse des électriciens et l'Union des centrales suisses d'électricité publient, pour ı go6, la statistique des stations centrales proprement dites fournissant du courant à des tiers. Celte statistique comparte des renseignements détall lés concernant le système, l'étendue et l'exploitation des diverses centrales.

Le: nombre tonal des stations est de $49 \%$, dont 245 ontét des stations génératrices particulières; 3 r reçớtvent de pénergte d'autres stations, soif en permanence, sót temporairement, pour ventr en arde a leurs propres installations ; 10 reçolvent 

centrale, et pour celle du rapport : Puissance à courants triphasés à puissance à courant continu à la sous-station, une même valeur de 84 pour 100 , correspondant à la puissance maxima de $200 \mathrm{kw}$. Grâze à un réglage convenable de l'excitation, le facteur de puissance put être fait égal à l'unité.

Près d'un an et demi après cet essai, la station centrale fut transformée et le courant continu fut remplacé par des courants triphasés; naturellement le groupe moteur générateur fut supprimé, et le moteur synchrone de la station réceptrice fut relié à la distribution générale à courants triphasés.

De nouveaux essais de rendement furent faits, mais on ne trouva plus qu'un rendement de 79 pour soo à pleine charge, en même temps le facteur de puissance ne put être amené égal à l'unité, il oscilla entre 0,9 I et 0,98 .

Comme, en somme, il ny avait de supprimé que le groupe moteur générateur de la station centrale, on remit ce dernier en mouvement, on fịt de nouveaux essais à la station réceptrice et on retrouva le rendement primitif de 84 pour Ioo, il ny avait donc pas eu d'erreur de mesures et l'anomalie constatée devait avoir d'autres causes.

Une enquête minutieuse ne tarda pas à donner la clef du mystèı : les alternateurs conduits par les moteurs à vapeur présentaient trois encoches par pôle et par phase, tandis que l'alternateur du moteur générateur aussi bien que-le moteur synchrone n'avaient que deux encoches par pôle et par phase.

On détermin 1 la courbe de la force électromotrice à vide de l'alternateur à vapeur et on la compara à celle du moteur synchrone, la différence était manifeste et, en les superposant, on vit qu'en retranchart des ordonnées de la première les ordonnées de la seconde, il restait une sinuscide à fréquence triple. L'explication du phénomène était dès lors facile : quand les machines étaient chargées, l'harmonique 3 du courant maintenait un courant de cette fréquence ( 150 par secondé) dans les enroulements; étant donné la self-induction élevée, ces courants étaient faibles, mais les courants de Foucault et les pertes par hystérésies dans le fer, qui augmentent très vite avec la fréquence, étaient la cause de la diminution de rendement observéc, ainsi que du plus faible facteur de puissance constaté.

\section{Une curieuse économie}

Nous découpons l'information suivante dans une publication d'un de nos confrères électriciens, généralement assez sérieux. Nous nous permettons seulement dy ajouter la ligne de la fin.

(En visitant les papeteries de Lancey, le berceau de la houille blanche, fondées par Aristide Bergès, et où est également installée l'usiae d'éclairage de la vallée du Grésivaudan, nous avons été étonnés de voir en mouvement toutes les transmissions, un jour de fête où les ouvriers ne travaillaient pas, et pendant le non-fonctionnement de l'usine électrique qui n'est mise en marche qu'à la tombée du jour.

“L'explication qui nous a été donnée est qu'il y a économie dans la fabrique, actionnée par les moteurs hydrauliques, à faire tourner tout le temps la transmission, les frais de graissage et de surveillance étant moins élevés que les réparations nécessitées par la dégradation des courroies par les rats, lorsque les transmissions sont arrêtées. 》

Se non è vero, è bene trovato.

\section{BIBLIOGRAPHIE:}

Recueil de types de ponts pour routes en béton de ciment armé, par $\mathrm{MM}$. de TEDEsco, ingenieur des arts et manufacture's et V. Fonestier. ingéuieur dis arts et métiers; I vol. et I atlas, de la collection Lechalas, Paris, Baudry.

Quand il ouvre pour la première fois un livre de cette espèce, qui s'annonce comme devant lui donner avec rapidité et précision les renseignements dont il a un besoin urgent, le lecteur va tout de suite au côté positif de l'ouvrage. Il s'embarrasse peu de la justification d.s théories, il a sur ce chapitre, fait crédit à son auteur en l'achetant; mais: ce qui lui importe par-dessus tout, c'est de savoir si le conseil qu'il va recevoir, répondra bien aux exigences industrielles, legnles, economiques, auxquelles il doit faire face et si l'appiication sera facile et éclairée d'exemples bien choisis..

Ceux qui useront du livre de MM. de Tedesco et Forestier, pourrontle faire en toute tranquillité d'esprit.
Les auteurs sont partis de la circulaire ministérielle du 20 octobre I 906 et des commentaires dont le Minis!re des tr.vaux publics et le Conseil des ponts et chaussées l'ont accompagnée. Dans une préface nette et concise, où $1 \mathrm{~s}$ expliquent clairement comment ils ont compris la tâche qu'ils se sont donné, surtout pour satisfaire à l'esprit des documents officiels, quand ceux-ci donnent intentionnellement à l'ingénieur exécutant une marge que l'état actuel de notre science en fuit de báton armé motive surabondamment.

Ce. faisant, MM. de Tedesco et Forestier auront contribué pour leur part à faire avancer cette science, et ils se sont donné d'avance comme auxiliaires tous ceux qui seront amenés à suivre leurs consells.

Le lecteur sera don: assuré de faire avec eux, une cuvre irréprochable, au point de vue scientifique comme au point de vue légal, point fort important.

Ce travail lui sera très facile.

Au chapitre I, page $5 \mathrm{r}$, il prendra connaissance du choix des unités. - Choix rationnel et très pratique.

Aux chapitres III 'et IV; il trouvera l'explication schématique de son cas ; puis, dans la deuxième partie. depuis le chapitre V jusqu'au chapitre XII, tous lis exemples numériques souhaitables pour les types de ponts de $4 \mathrm{~m}$. à $30 \mathrm{~m}$. De la sorte, il n'aura nul embarras pour résoudre son cas particulier si, comme il est fréquent, il ne rentre pas dans un type traitś, et il aura de plus un exemple clair lui permettant aussi de présenter son projet avec méthode, clarté et rapidité.

Un atlas accompagne le volume.

Dans cet ordre d'idées, nous avons, au mois de septembre r 905 , rendu compte, ici même, d'un ouvrage analogue de $M$. Maurice Kœihlin.

Ce qu 2 nous avons dit alors de l'utilité de son livre, s'applique pour celui de M.M. de Tedesco et Forestier et nous dispense d'insister autrement que pour montrer à nos confrères, que ces deux ouvrages se complètent, à l'effet de mettre entre leurs mains les outils avec lesquels ils peuvent satisfaire à toutes leurs exigences professionnelles en la matière spéciale.

Les éloges que nous donnions à la maison Baudry doivent être répétés dans les mèmes termes ; mais, malheureusement, la critique que nous faisions aux typographes, en ce qui a trait à la saine ortbographe des nombres, doit être répéte mot pour mot. Nous y renvoyons qui de droit; pour notre compte, nous ne nous lasserons pas de répéter que le point séparatif des tranches de trois chiffres d'un nombre est une faute d'orthographe. Un blanc'est nécessaire et suffisant. Malgré cette petite réserve, beau livre.

\section{Commandant Auderrand.}

Service d'études des grandes forces hydrauliques dans la région des Alpes. - Comptes-rendus des travaux de MiM. R. TAVERNIER et R. DE LA Brosse, ingénieurs en chef des ponts et chaussées. Documents ofticiels publiés par les Annales du Ministère de l'Agriculture. 2 volumes grand in $-\bar{\delta}^{\circ}$; prix broché, i 5 fr.; franco, 16 tr., chez Gratier el Rey, Grenoble.

Ces deux volumes contiennent le compte-rendu des travaux exécutés par les deux éminents ingénieurs qui ont été chargés par le Ministère de l'Agriculture du service d'études des grandes forces hydrauliques des Alpes. Nous nous proposons d'en faire une étude détaillée, sous forme d'art.cle, dans un prochain numéro; nous nous contenterons de signaler dès à présent les très intéressantes carteś hydrologiques des bassins de l'Arve, de i'Isère, de la Durance qui accompagnent ces deux ouvrages que tous ceux qui s'intéressent à l'aménagement des chutes d'eau consulteront avec le plus grand intérêt et pour leur plus grand profit. E.-F. Cồté.

\section{LIVRES NOUVEAUX EN FRANCE ET A L'ÉTRANGER}

Les distributions publiques d'énergie électrique en France, S. A. MONTP'Eltiar. In $-40,25$ franes.

Les courants alternatifs mainstriels et leurs applications. R. Berger. In- $-8^{\circ}, 5$ francs.

Préservalion des bois contre la pourriture par. le sol, les champignons et les insectes, HenRY. In-8o, 4 francs.

Carte des stations centrales d'électricité en Suisse, 4. fr. 50.

How to use Water Power. ChateEY. In-80, 4 fr. 25.

Praclical hydraulic. Housden. In-8o, $5 \mathrm{fr}$. 75.

Ansbau von Wasserkraften. KоенN. In-8o, 38 fr. 75.

Nota. - Nos lecteurs pourront se procurer ces lirres, chez Gratiek et REy, à Grenoble, éditeurs de La. Houille Blanche.

\section{L'Imprimeur-Gérant : P. L.EGENDRE.}

